\title{
TEST OF A RADIO-FREQUENCY QUADRUPOLE COLD MODEL
}

\author{
G. V. Lamanna", INFN-Bari, A. Lombardi, A. Pisent, INFN-LNL
}

\begin{abstract}
In the framework of TRASCO Project (the Italian feasibility study for a waste transmutation linac) we have built and tested an aluminium model of a segmented resonantly coupled Radio-Frequency Quadrupole (RFQ), in order to determine the parameters of a $5 \mathrm{MeV}$ and $30 \mathrm{~mA}$ proton beam RFQ. Bead perturbation measurements are performed, and the field stabilization respect to small perturbations of quadrupole fundamental mode is studied. A comparison between experimental results and a theoretical model is also reported.
\end{abstract}

\section{INTRODUCTION}

The possibility to design an RFQ long respect to the RF wavelength is very important for the feasibility of linacs with different applications, from the high power $\mathrm{CW}$ linacs for neutron production to the low power high frequency linacs proposed for hadrotherapy [1]. In particular INFN has been funded for the study of the critical parts of a waste transmutation linac (TRASCO project). In this framework the field stabilization of a 7 $\mathrm{m}$ long $352 \mathrm{MHz}$ RFQ has been studied, using the LANL resonant coupling technique.

We looked for an approach able to give a physical insight of the system and fast results to be compared with MAFIA simulations and field measurements; the first results, achieved by an equivalent lumped circuit [2], have been improved by the implementation of a transmission line analysis [3]. Moreover an aluminium model has been built and we are now testing on it the RFQ tuning procedure.

In this paper we describe the aluminium model, we resume the main results of the transmission line analysis and we show the first results of the bead pull measurements.

\section{THE MODEL}

The aluminium model had to be at the same time similar to the actual $\mathrm{Cu}$ construction, flexible, easy to modify and with a reasonable cost. We required very good mechanical tolerances to allow an easy determination of quadrupole and dipole modes. Mechanical errors can be introduced on purpose so to verify RF field tolerances.
The body of each segment has a "sandwich" configuration, with four parts bolted and pined to $20 \mu \mathrm{m}$ alignment tolerance. The end cells undercuts electrode dimensions were built with some extra material; during tests they were easily dismounted and machined (Fig.1).

Each segment is supported in three points so to permit fine mechanical alignment. Overall mechanical tolerance is about $50 \mu \mathrm{m}$. The first and the last segment can slide respect to the central one in order to disassemble the coupling cell and vary its configuration preserving the alignment.

The RFQ consists of three $1 \mathrm{~m}$ long segments. Vanes are unmodulated with an average aperture radius of 5 $\mathrm{mm}$. The resonant frequency of $352 \mathrm{MHz}$ is provided by 36 tuners, i. e. 12 screws per segment having $20 \mathrm{~mm}$ diameter, with a penetration of $13 \mathrm{~mm}$ in nominal position. Eight antennas are available in each segment for powering and measuring.

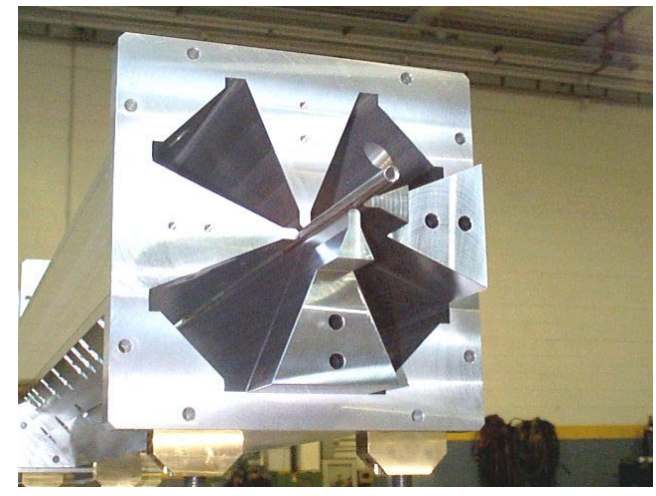

Figure 1 View of coupling cell electrodes

\section{RFQ THEORETICAL MODEL}

A four vanes RFQ is based on $\mathrm{TE}_{21}$ modes, and the vane voltage transformation after a segment of length $\lambda$ is:

$$
\left[\begin{array}{c}
V \\
V^{\prime}
\end{array}\right]_{\lambda}=\left[\begin{array}{cc}
\cos k \lambda & \frac{1}{k} \sin k \lambda \\
-k \sin k \lambda & \cos k \lambda
\end{array}\right]\left[\begin{array}{c}
V \\
V^{\prime}
\end{array}\right]_{0}=M_{r f q}\left[\begin{array}{c}
V \\
V^{\prime}
\end{array}\right]_{0}
$$

with $\quad k=\frac{2 \pi \sqrt{x-1}}{\lambda}, x=\frac{\omega^{2}}{\omega_{0}^{2}}$ and $\omega_{0}=\frac{2 \pi c}{\lambda} \quad$ cut-off frequency. Note that below cut-off frequency $k$ is imaginary, but if we define the trigonometric functions in the complex field the matrix elements are real.

\footnotetext{
${ }^{\#}$ Email: giuseppe.lamanna@ba.infn.it
} 
The coupling cell can be modeled by the capacitance $\mathrm{C}_{\mathrm{a}}$ in the gap between each couple of electrode terminations, i.e. with the transformation:

$$
\left[\begin{array}{c}
V \\
V^{\prime}
\end{array}\right]_{+}=\left[\begin{array}{cc}
1 & -\frac{\lambda \beta}{x} \\
0 & 1
\end{array}\right]\left[\begin{array}{l}
V \\
V
\end{array}\right]_{\lambda}=M_{g a p}\left[\begin{array}{c}
V \\
V
\end{array}\right]_{\lambda},
$$

with: $\beta=\frac{\lambda^{2}}{4 \pi^{2}} \frac{C}{C_{a} \lambda}$ and $C$ capacitance per unit length of the quadrupole vane tips per quadrant (approximately 30 $\mathrm{pF} / \mathrm{m})$. The Floquet analysis gives the dispersion relation:

$$
\cos \alpha=f(x)=\frac{t r M_{r f q} M_{g a p}}{2}=\cos k \lambda+\frac{k \lambda \beta}{2 x} \sin k \lambda
$$

with $\alpha$ phase advance per period. For $\beta=0$ the RFQ segments are directly connected and only modes above cut-off frequency exist, while otherwise different bands are present, corresponding to $|f(x)| \leq 1$. The fundamental mode $x=1$ of the resonator can be stabilized if around this mode we have the confluence of two bands with lower and higher frequencies; since $f(1)=1$, this happens when $f(x)$ has a maximum at that frequency, i.e. when $f^{\prime}(1)=0$ or $\beta=1$.

The same dispersion relation can be calculated for $\mathrm{TE}_{11}$ modes, normalizing the frequency for the appropriate cut-off frequency $\omega_{\mathrm{d}}$ and using the coupling parameter:

$$
\beta_{d}=\beta \frac{1}{2}\left[\frac{\omega_{0}}{\omega_{d}}\right]^{4}
$$

The results of this model are compared with results in literature in ref [3].

This simple model can be refined introducing the effect of the end cells and by considering the finite number $\mathrm{N}_{\mathrm{RFQ}}$ of segments that compose our resonator.

Indeed the boundary condition $V^{\prime}=0$ at the two extremes, that for a TM mode corresponds to a metallic boundary, for a TE mode is obtained by terminating the transmission line on a resonant load, called end-cell (EC), with admittance $1 / i \omega L_{E C}+i \omega C_{E C}$. The associated linear transformation is:

$$
\left[\begin{array}{l}
V \\
V^{\prime}
\end{array}\right]_{0}=\left[\begin{array}{cc}
1 & 0 \\
\xi(\kappa-x) & 1
\end{array}\right]\left[\begin{array}{c}
V \\
V^{\prime}
\end{array}\right]_{-}=M_{E C}\left[\begin{array}{c}
V \\
V^{\prime}
\end{array}\right]_{-}
$$

with the parameters: $\xi=\frac{4 \pi^{2}}{\lambda^{2}} \frac{C_{E C}}{C}$ and $\kappa=\left(L_{E C} C_{E C} \omega_{0}^{2}\right)^{-1}$.

The array with - subscript corresponds to the ideal termination values. The two parameters $\kappa$ and $\xi$ correspond respectively to the resonant frequency of the $\mathrm{EC}$ and to the relative weight of the EC respect to the
RFQ body in terms of stored energy. In a real RFQ $(\xi \neq 0)$ the boundary condition $V^{\prime}=0$ holds only if the ECs are perfectly tuned $(\kappa=1)$, while all the other modes see a termination different from the ideal one. In a single RFQ segment, if the EC is tuned too high in frequency, $V^{\prime}>0$ and the voltage in the EC is lower then in inside the RFQ.

We can therefore calculate the transfer matrix for $N_{R F Q}$ segments:

$$
M=\left(M_{E C} M_{r f q} M_{E C} M_{g a p}\right)^{N_{R F Q}-1} M_{E C} M_{r f q} M_{E C}
$$

and the modes are defined by the condition

$$
\left[\begin{array}{c}
V_{\text {out }} \\
0
\end{array}\right]_{\text {out }}=M\left[\begin{array}{c}
V_{\text {in }} \\
0
\end{array}\right]_{\text {in }} \quad \text { i.e. } \quad M_{2,1}=0
$$

corresponding to the condition at $V^{\prime}=0$ the two extremes. In section 3 we shall compare the zeros of $M_{21}$ with the measured mode frequencies.

\section{EXPERIMENTAL RESULTS}

The experimental apparatus (Fig. 2) consists of the bead pulling system (a dielectric bead of $4 \mathrm{~mm}$ radius driven by a cc motor by means of a kevlar thread of $0.25 \mathrm{~mm}$ diameter), a Network analyzer (NA) HP 4185A and a trigger unit driving NA and cc motor. The bead touches a couple of electrodes so to have the best reproducibility. Phase measurement is performed with a NA resolution bandwidth $R W=300 \mathrm{~Hz}$, resulting in a phase error of $0.14 \mathrm{deg}$.

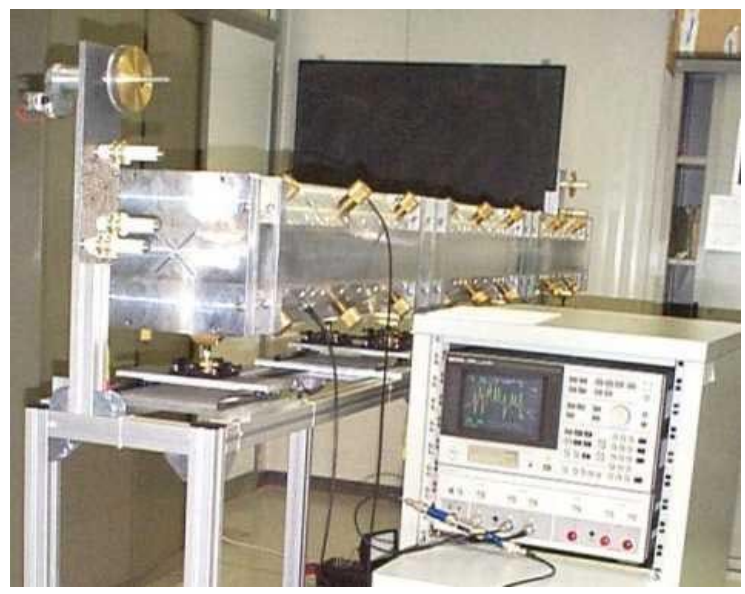

Figure 2 View of the experimental apparatus

For a dielectric bead moving inside the RFQ the electric field $E(z)$ at bead location $z$ and the variation $\Delta \phi(z)$ of the output phase respect to when the bead is outside the RFQ (when exciting the system at the unperturbed resonance frequency) are related (for small $\Delta \phi)$ by the equation: 


$$
\frac{E(z)^{2}-E_{0}^{2}}{E_{0}^{2}}=\frac{\Delta \phi(z)-\Delta \phi_{0}}{\Delta \phi_{0}}
$$

where $\Delta \phi_{0}$ is the phase variation corresponding to the maximum field $\mathrm{E}_{0}$ for a given measurement.

Therefore the phase shift gives a relative measurement of the electric field, with the advantage to be unaffected by temperature variation since a single phase plot is taken in about $30 \mathrm{sec}$. Moreover no computer control is needed and phase plot, i.e. E-field variation along the structure, is shown on the NA screen. The flatness $F$ (maximum of $\Delta E / E$ ) is easily evaluated.

Firstly we have tuned end cells by cutting the electrode ends in order to flatten the field (making $k=1$ ) in each 1 $\mathrm{m}$ segment. In $\mathrm{Tab} 1$ the comparison between the frequencies before and after tuning is reported. The frequency is slightly different from $v=350.7 \mathrm{MHz}$ and the tuners insertion turns out to be not exactly $13 \mathrm{~mm}$.

Table 1: Segment frequencies comparison

\begin{tabular}{|c|c|c|}
\hline Segment & $\begin{array}{c}\text { Before tuning } \\
\text { (MHz) }\end{array}$ & $\begin{array}{c}\text { After tuning } \\
\text { (MHz) }\end{array}$ \\
\hline 1st & 350.625 & 352.046 \\
\hline 2nd & 350.525 & 352.040 \\
\hline 3rd & 350.615 & 352.045 \\
\hline
\end{tabular}

In Fig. 3 phase plots of the tree tuned segments are shown $(F \approx 2.4 \%)$. If we consider the interval between the two arrows, corresponding to the region between the end cell electrodes, $F \approx 0.55 \%$. The tuners are situated in correspondence of the valleys.

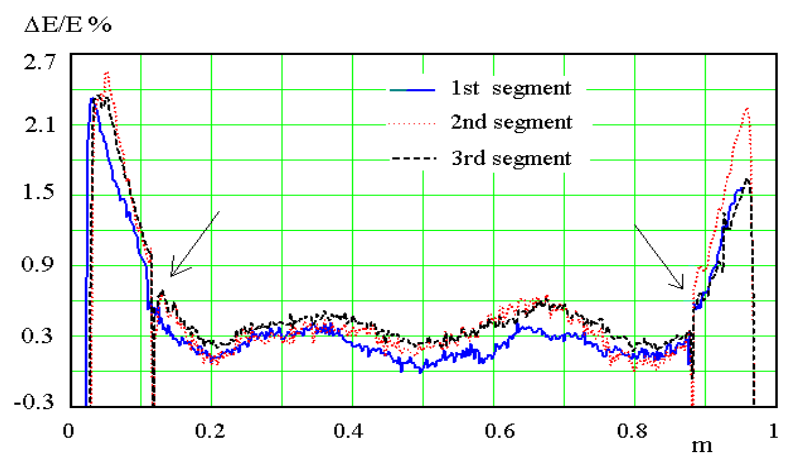

Figure 3: Phase plot of each segments

The phase plot for the three segments connected together and separated by 2 coupling cell is shown in Fig.4. The tuning of the coupling cells have been accomplished by cutting undercuts and working the upper part of coupling cells electrodes. The first action causes a change in the capacitance, so to get $\beta=1$, the second an inductance variation (making $\kappa=1$ ). The operating frequency is recuperated acting on the tuners; the achieved flatness (end cells excluded) is $F \approx 3.1 \%$.

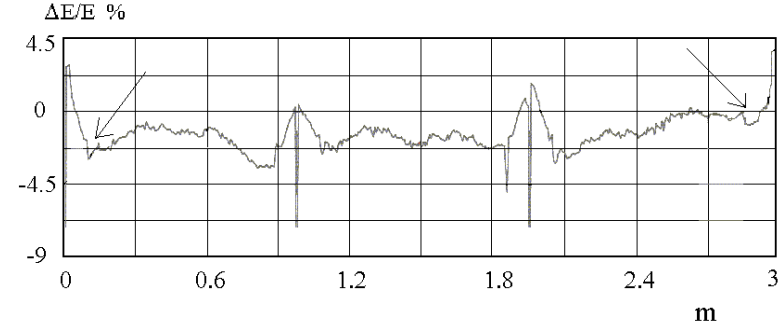

Figure 4: three segments with two coupling cells

The comparison between experimental frequency spectrum and the theoretical one is shown in Fig.5. The spectra are in quite good agreement with the exception of the last two modes, that are close in frequency with a possible mix between dipoles and quadrupoles.

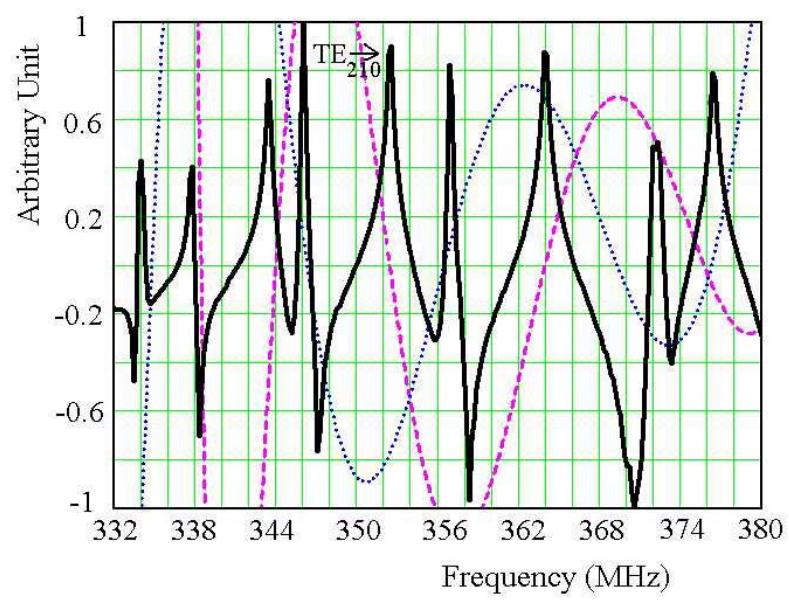

Figure 5: Comparison between experimental (black line) ant theoretical spectra obtained from the zeros of $\mathrm{M}_{21}$ of the dipole mode (dotted bleu line) and the quadrupole mode (magenta dashed line)

The future work will be devoted to the modal analysis of the beadpull data in order to set up an automatic procedure to tune the RFQ up to $1-2 \%$ E-field error.

\section{ACNOWLEDGMENTS}

The authors thank S. Marigo, M. Negrato, and V. Valentino for their help in assembling the set-up.

\section{REFERENCES}

[1]L.M.Young "An 8-meter long coupled cavity RFQ linac" Proceedings of the 1994 International Linac Conference, Tsukuba, Japan, p.178 (1994).

[2]A.Pisent ,R. Celentano, R. Zennaro "Equivalent lumped circuit study for the field stabilization of a long four-vanes RFQ". Presented at 1998 linac conference.

[3] A. Pisent "Transmission line analysis of coupled RFQ's" TRASCO note $01 / 99$ (1999). 